Nottingham, Gwynedd and Neath Port Talbot). Eligible individuals were randomly selected from primary care trusts to health board registries. Assessment consists on multi-dimensional aspects of health, collection of saliva samples, and permission for review of general practice medical records. Those, who consent, will be flagged with the National Health Service central register to provide details of the date and cause of death.

Results Collection of data are still ongoing and we will present what was collected up until December 2011.

Conclusions CFAS II, in combination with its parent study will address key questions about health, diseases, associated disability, policy projections across generations of older people, who will reach the age of greatest frailty in the 2020s when the peak in numbers of 85 and over is expected.

\section{P1-245 CAMBRIDGE CENTRE FOR AGEING AND NEUROSCIENCE (CAMCAN) STUDY PROTOCOL}

doi:10.1136/jech.2011.142976e.37

T Minett, ${ }^{*}$ on behalf of CamCAN team and investigators. Cambridge University, Cambridge, UK

Background As the world population is ageing, and ageing is often stereotyped as a time of mental restriction and inflexibility, individuals make flexible use of available resources, including recruiting regions and other cognitive processes. Our aim is to identify what determines successful ageing across the adult lifespan into old age of cognitive abilities such as memory, attention, emotion, language and action.

Methods A population-based cohort of 3000 adults, aged 18+, will be recruited with demographic and basic cognitive assessments. Of these, 700, aged 18-87 with 100 per decile, will be selected for comprising structural and functional neuroimaging [MRI and magnetoencephalography (MEG)] and neuropsychological tests. We will measure neural integrity and integration across cortical regions. On a subset of 280 adults further investigations will use functional MRI, MEG and electroencephalogram, and further behavioural testing. Formal statistical models will be used to examine the changes that occur with healthy ageing, and the reorganisation in terms of strategies and structures invoked to compensate for them. This approach offers hypothesis-driven insights into healthy ageing that are relevant to the general population.

Results Collection of data started in Jan-11, with the initial cohort taking 2 years to recruit and a further 3 years for all detailed investigations.

Conclusions Our research will generate a unique resource of neuroimaging and cognitive measures about change across the adult lifespan. Our analysis will help us to identify what characterises older adults with preserved performance and how normal ageing differs from pathological ageing in conditions such as Alzheimer's disease.

\section{P1-246 NATIONAL PREVALENCE AND RISK BEHAVIOURS OF CHLAMYDIA TRACHOMATIS INFECTION AMONG PREGNANT WOMEN AGED 15 TO 24 IN BRAZIL}

doi:10.1136/jech.2011.142976e.38

${ }^{1} \mathrm{~A}$ Miranda, ${ }^{* 1,2} \mathrm{~V}$ Pinto, ${ }^{1} \mathrm{C}$ Baroni, ${ }^{1} \mathrm{~L}$ Stringari, ${ }^{3} \mathrm{C}$ Szwarcwald. ${ }^{1}$ Universidade Federal do Espirito Santo, Vitoria, Espirito Santo, Brazil; ${ }^{2}$ Programa Nacional DST/AIDS, Ministério da Saude, Brasília, Distrito Federal, Brazil; ${ }^{3}$ Fundação Oswaldo Cruz, Rio de Janeiro, Brazil

Introduction Chlamydia trachomatis (CT) is a sexually transmitted infection having repercussions on reproductive health and impact on the fetus.
Purpose To estimate Chlamydia trachomatis prevalence and risk factors in pregnant women aged 15 to 24 in Brazil.

Methods A national cross-sectional study among pregnant women attending Brazilian public maternity units in 2009. The participants were screened for CT and Neisseria gonorrhoeae, using polymerase chain reaction in urine, and also answered a questionnaire including demographic, behavioural and clinical data.

Results A total of 2071 (86.3\%) of 2400 pregnant women selected took part in the study. Their mean age was 20.2 years (SD 2.7). Chlamydia and Gonococcus infection prevalence was, respectively, $9.8 \%$ (95\% CI 8.5 to 11.1 ) and $1.0 \%$ (95\% CI $0.6 \%$ to $1.4 \%)$. Four per cent of women infected with Chlamydia also had simultaneous Gonococcus infection. CT associated factors were being aged between 15 and 19 [OR=1.6 (95\% CI 1.15 to 2.17)], first sex intercourse before 15 years of age $[\mathrm{OR}=1.4 \quad(95 \% \mathrm{CI} 1.04$ to 6.24)], having had more than one sex partner in their lives $[\mathrm{OR}=1.6(95 \% \mathrm{CI} 1.13$ to 2.26$)]$, having undergone oncotic cytology more than 1 year ago $[\mathrm{OR}=1.5$ (95\% CI 1.08 to 2.05$)]$ and having had gonococcal infection $[\mathrm{OR}=7.6$ (95\% CI 3.05 to 19.08)].

Conclusions Health programmes need to pay attention to the need to screen for easily curable sexually transmitted infections, such as Chlamydia trachomatis, in populations that are more vulnerable and at greater risk. This study suggests that CT diagnosis should be included as part of the antenatal routine of young pregnant women, since infection prevalence found in this group was high.

\section{P1-247 CEREBROVASCULAR DISEASE IN 48 COUNTRIES: SECULAR TRENDS IN MORTALITY 1950-2005}

doi:10.1136/jech.2011.142976e.39

${ }^{1} \mathrm{M}$ Mirzaei, ${ }^{*} \mathrm{R}$ Taylor, ${ }^{2} \mathrm{~S}$ Truswell, ${ }^{4} \mathrm{~A}$ Pages, ${ }^{4} \mathrm{~K}$ Arnett, ${ }^{2} \mathrm{~S}$ Leeder. ${ }^{1}$ Shahid Sdoughi University of Medical Sciences, Yazd, Iran; ${ }^{2}$ The University of Sydney, Sydney, Australia; ${ }^{3}$ The University of New South Wales, Sydney, Australia; ${ }^{4}$ The University of Queensland, Brisbane, Australia

Cerebrovascular disease (stroke) is the second cause of death and among the top five causes of morbidity in many developed and developing countries. The coincidence of trends of stroke and coronary heart disease mortalities is of question in different countries. This study aims to investigate patterns of increase and decrease of stroke mortality in 48 different countries. The mortality curves of stroke for 48 countries that had reliable data and met other selection criteria were examined using age-standardised death rates for 35-74 years from the WHO. Annual male mortality rates for individual countries from 1950 to 2005 were plotted and a table and graph were used to classify countries by magnitude, pattern and timing of stroke mortality. The natural history of stroke epidemics varies markedly among countries. Different stroke patterns are distinguishable; including "declining" (since the inception of data or 1950), "rise and fall", "rising" (first part of epidemic), and "flat" (no epidemic yet). Further, epidemic peaks were higher in Asia, in particular Japan at 433/105, the former Soviet states at 388/105 and East Europe at 301/105 and lowest in Canada and Australia at 29/105. The different dates of mortality downturn could reflect the times when pharmaceutical treatment of hypertension started to be effective in sufficient numbers of the high risk population and/or there were significant changes in salt consumption. This could be translated to policy interventions for stroke control in countries with rising trend of the disease. 